\title{
Discrete element investigation of the asymptotic behaviour of granular materials
}

\author{
D. Mašín \\ Faculty of Science, Charles University in Prague, Czech Republic \\ e-mail: masin@natur.cuni.cz
}

Summary. The concept of the asymptotic behaviour of particulate materials is described, including its enhancement considering asymptotic states in extension. A 3D discrete element model with permanent elastic spherical particles is set up. The numerical sample is stretched from different initial states, and the influence of the strain rate direction on the final state is studied. Asymptotic behaviour is clearly observed. Existence of extension asymptotic states is demonstrated, and the notion of normal extension line is introduced.

Keywords: asymptotic behaviour, critical state, discrete element method, particle crushing, sand.

\section{Introduction}

Asymptotic behaviour is one of the most striking features in the behaviour of granular materials. Specific asymptotic states have been known since the early days of soil mechanics. Casagrande [1] observed critical state behaviour of soils - a particular asymptotic state related to constant volume shearing. Schofield and Wroth [8] combined the existence of critical states and compression asymptotic states (revealed in normal compression behaviour) into a unified framework of critical state soil mechanics. More generally, Gudehus et al. [5] understood asymptotic states to be attractors in the behaviour of granular materials, which are independent of the initial state. They proposed that each direction of strain rate with a volume decrease is uniquely linked to a particular asymptotic stress ratio and a particular path in the mean stress vs. void ratio plane (normal compression line). More recently, Gudehus [3] and Gudehus and Mašín [4] have extended the asymptotic state concept into the volume increase (extension) regime.

In this contribution, we present a discrete element study aimed at understanding of the asymptotic behaviour of granular materials. Only the main outcomes of an 
extensive study are presented due to limited space; more details are given in Mašín [7].

\section{Asymptotic state framework}

Asymptotic states are defined as that states reached after a sufficiently long proportional stretching, i.e. stretching with a constant direction of the strain rate. Conceptual representation of asymptotic states has been proposed by Gudehus [3] and Gudehus and Mašín [4]. In this work, we focus on axisymmetric stress and deformation states, where the strain rate tensor is fully characterised by axial $\dot{\varepsilon}_{a}$ and radial $\dot{\varepsilon}_{r}$ components. Similarly, the stress tensor is given by $\sigma_{a}$ (axial stress) and $\sigma_{r}$ (radial stress). The strain rate direction may be characterised by an angle $\psi_{\dot{\varepsilon}}$ (see Figure 1a), and the stress obliquity is quantified by the angle $\psi_{\sigma}$ (Figure 1b).

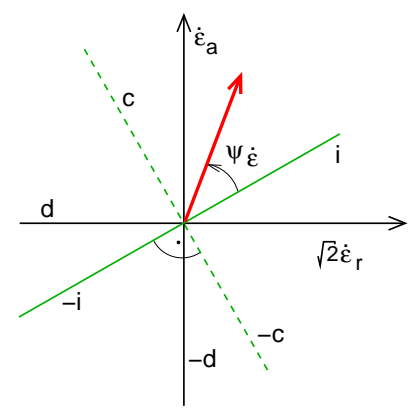

(a)

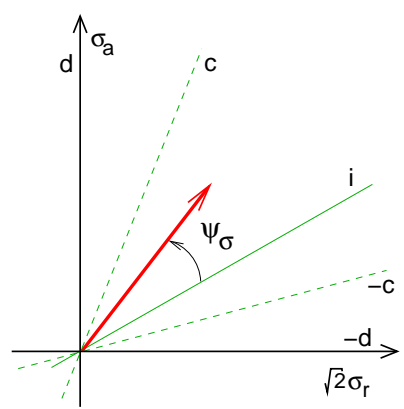

(b)

Fig. 1 Definition of angles $\psi_{\dot{\varepsilon}}$ and $\psi_{\sigma}$ [4]. "i" denotes the isotropic direction, "c" denotes the isochoric (constant volume) direction, and "d" denotes the theoretical limit for asymptotic state behaviour.

According to the current understanding of the asymptotic behaviour of a granular assembly, proportional deformation (constant $\psi_{\dot{\varepsilon}}$ ) will ultimately lead to an asymptotic state characterised by a constant $\psi_{\sigma}$. Each of the asymptotic states also has a unique trace in the mean stress $p$ vs. void ratio $e$ plane. Asymptotic states in this plane are traditionally denoted as normal compression lines. All normal compression lines are bound between isotropic normal compression line, attributed to $\psi_{\dot{\varepsilon}(i)}$, and the critical state line, linked with $\psi_{\dot{\varepsilon}( \pm c)}$ (see Fig. 1 for definition of $i$ and $\pm c$ directions).

As suggested by Gudehus [3] (Chapters 2 and 3) and Gudehus and Mašín [4], asymptotic states can also be reached after proportional stretching along extension (volume increase) paths. The stretching directions that lead to extension asymptotic states are depicted in Figure 2. Limiting values of $\psi_{\dot{\varepsilon}}$ and $\psi_{\sigma}$ are denoted with indices 'd' (asymptotic $\sigma_{r}=0$ ) and '-d' (asymptotic $\sigma_{a}=0$ ) [4]. Each extension 
asymptotic state is also associated with its trace in the mean stress vs. void ratio plane. In the following, we denote these traces as normal extension lines (adopting a parallel with the well-known notion of normal compression lines).

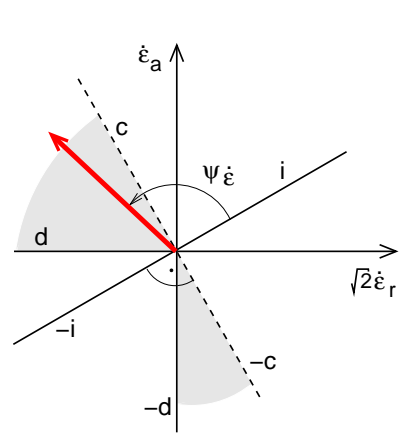

(a)

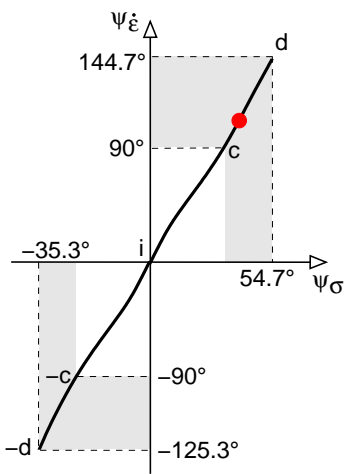

(b)

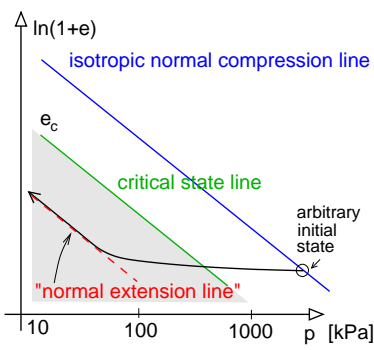

(c)

Fig. 2 Graphical representation of extension asymptotic states.

\section{Discrete element model of the asymptotic behaviour}

In order to model the granular assembly, the open-source 3D discrete element software Yade [9] was used. This software utilises the DEM formulation given by Cundall and Strack [2]. We present the results for a specimen consisting of elastic spherical particles. In order to resemble a real granular material, particles of different sizes have been considered following the grain-size-distribution curve of a real sand. The sample consisted of 150000 spherical particles. The periodic cell was cubic, with the initial side length of $31 \mathrm{~mm}$. The specimen in its initial state is depicted in Figure 3.

The contact properties of the spherical particles were governed by a linear elastic perfectly plastic model without cohesion [2], which specifies the contact normal stiffness $k_{n}$, shear stiffness $k_{s}$ and friction angle $\varphi$. These parameters are calculated from the particle properties $E=500 \mathrm{MPa}, v=0.3$ and $\varphi=0.5 \mathrm{Rad}$. The prescribed particle density was $\rho_{s}=2650 \mathrm{~kg} / \mathrm{m}^{3}$ and acceleration due to gravity was zero. The cell boundaries were periodic and they were subjected to a constant velocity gradient $\nabla v$ (constant value of the Euler stretching tensor D). Axisymmetric conditions were applied, such that $D_{22}=D_{33}$ (subscripts 2 and 3 represent the horizontal directions, 1 the vertical direction). The angle $\psi_{\dot{\varepsilon}}$ can then be calculated by means of Figure 1(a). Local non-viscous damping has been used, with a damping coefficient $\chi=0.5$. 


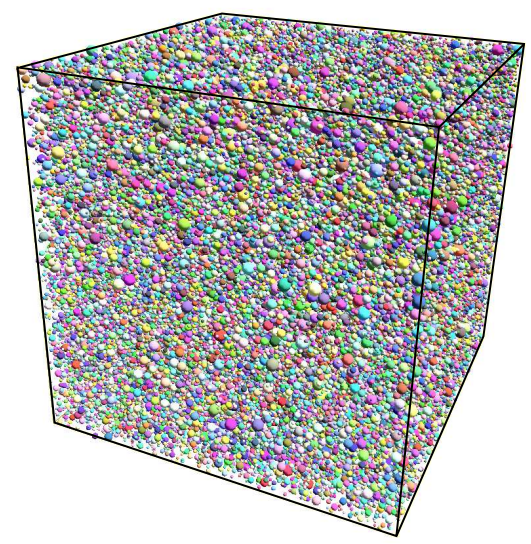

Fig. 3 Periodic cell used in the simulations, consisting of 150000 spherical particles.

\section{Modelling results}

Figure 4 shows results on a sample loaded uniformly along the path $\psi_{\dot{\varepsilon}}=0^{\circ}$, together with the results of a test where the loading direction was reversed several times to $\psi_{\dot{\varepsilon}}=180^{\circ}$. The uniformly loaded sample clearly defines an isotropic normal compression line, which is approximately linear in the $\ln p$ vs. $\ln (1+e)$ plane. The sample with the unloading-reloading cycles shows that the isotropic normal is asymptotically approached irrespective of the initial soil density (apart from the minor "overshooting" in the first reloading cycle, which may be attributed to inertia effects). The normal compression behaviour is observed on an assembly of elastic (non-crushable) spheres; it is thus not related to grain crushing. Instead, grain crushing may be regarded as an eventual consequence of stress concentration during loading along the normal compression line.

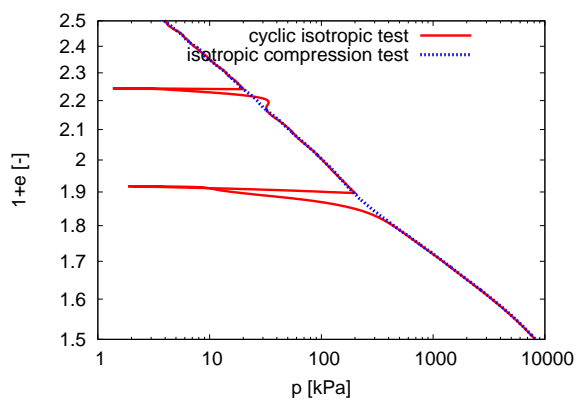

Fig. 4 Monotonous and cyclic (unloading-reloading) isotropic test results $\left(\psi_{\dot{\varepsilon}}=0^{\circ}\right.$ in loading and $\psi_{\dot{\varepsilon}}=180^{\circ}$ in unloading). 
Secondly, we study the constant volume asymptotic state $\left(\psi_{\dot{\varepsilon}}=90^{\circ}\right)$. Figure 5 shows the results of constant volume experiments on normally consolidated (NC) and overconsolidated (OC) samples. These tests are known as undrained triaxial tests in soil mechanics terminology. The samples were first loaded along the $\psi_{\dot{\varepsilon}}=0^{\circ}$ path up to the prescribed value of the mean stress. Then, the direction of loading was changed to $\psi_{\dot{\varepsilon}}=90^{\circ}$ and the NC samples were sheared until the asymptotic state was reached. The $\mathrm{OC}$ samples underwent $\psi_{\dot{\varepsilon}}=180^{\circ}$ unloading before shearing. Figure 5 shows results of the numerical experiments. Although the final states appear to align along a single curve in the $p$ vs. $\psi_{\sigma}$ plane, this curve is not represented by a constant value of $\psi_{\sigma}$. The final states in the $\ln (1+e)$ vs. $\ln p$ plane form a clearly defined critical state line. This line is approximately parallel to the isotropic normal compression line, apart from the larger stresses, where the results are influenced by the final compressibility of the grains and consequent non-negligible overlapping of particles. The asymptotic state is reached irrespective of the initial void ratio (NC and OC samples reach the same state finally).

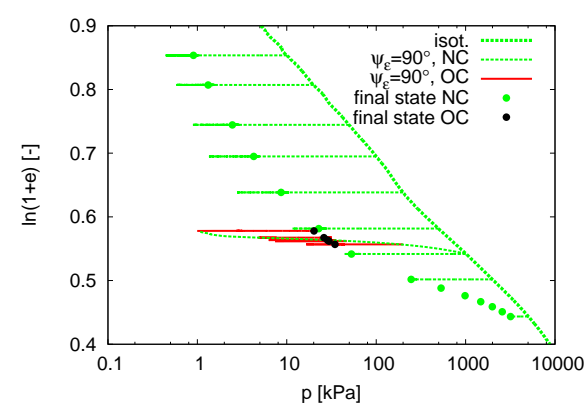

(a)

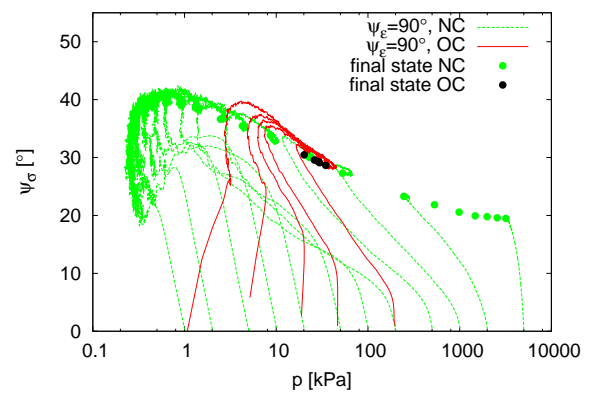

(b)

Fig. 5 Results of $\psi_{\dot{\varepsilon}}=90^{\circ}$ tests on normally consolidated and overconsolidated samples.

In the next set of simulations, we consider a shear test in which the stress path (rather than the strain path) direction is controlled. Namely, experiments with a constant $\sigma_{r}$ (drained triaxial tests) have been simulated. The same stretching rate $D_{11}$ as in the $\psi_{\dot{\varepsilon}}=90^{\circ}$ tests was imposed, and $D_{22}=D_{33}$ were controlled in such a way that $\sigma_{r}$ remained constant. After sufficiently long shearing, the specimens reached a state with constant $\sigma_{a}$ and constant $e$, therefore with constant $\psi_{\sigma}$ and with $\psi_{\dot{\varepsilon}}=90^{\circ}$ (see Figure 6). Tests on both normally consolidated and overconsolidated samples were considered. The asymptotic states reached by the NC and OC samples coincided for the given $\sigma_{r}$ (Figure 6). The asymptotic states reached in constant $\sigma_{r}$ tests coincided also with those from $\psi_{\dot{\varepsilon}}=90^{\circ}$ tests.

Apart from the asymptotic states in compression, asymptotic behaviour of samples stretched in extension $\left(\psi_{\dot{\varepsilon}}>90^{\circ}\right)$ has been investigated. Figure 7 shows the $\ln (1+e)$ vs. $\ln p$ response of these samples. Irrespective of the initial compression stress, all the samples reached well defined asymptotic states. These lines were proposed in Sec. 2 as "normal extension lines". Although they have probably not been 


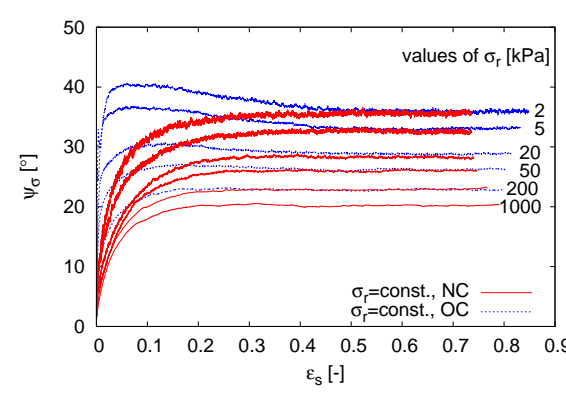

(a)

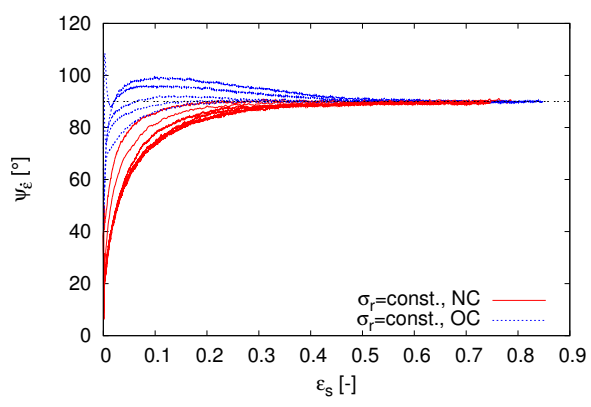

(b)

Fig. 6 Results of constant $\sigma_{r}$ tests on normally consolidated and overconsolidated samples.

observed in experiments yet, they were theoretically predicted and in this study they were confirmed using discrete element simulations.

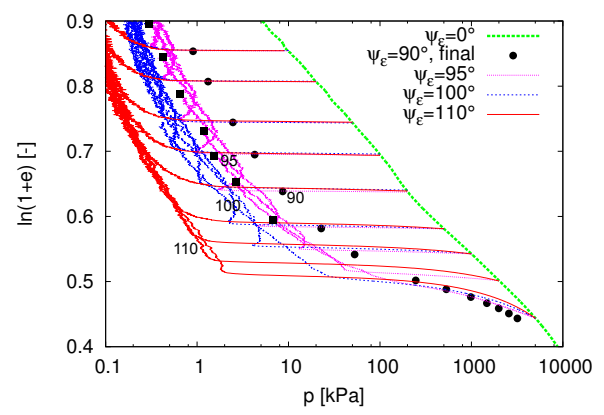

Fig. 7 Extension asymptotic states with $\psi_{\dot{\varepsilon}} \geq 90^{\circ}$ plotted in the $\ln (1+e)$ vs. $\log p$ plane, demonstrating existence of the so-called normal extension lines.

Figure 8 attempts to summarise the asymptotic states obtained in the described discrete element simulations for various angles of $\psi_{\dot{\varepsilon}}$. Several properties of the observed asymptotic states agree with the concept described in Sec. 2. There are, however, also clear deviations from this concept. As expected, increasing $\psi_{\dot{\varepsilon}}$ leads to an increase of the asymptotic stress ratio $\psi_{\sigma}$. Contrary to the theory and experimental observations, however, the asymptotic $\psi_{\sigma}$ is for the given $\psi_{\dot{\varepsilon}}$ not constant, but it depends on the mean stress level (Fig. 8(a)). This dependency is likely to be caused by rate effects, which can be expressed in terms of inertial number $I$ [6]; see Mašín [7] for more detailed discussion. Asymptotic states were uniquely defined also in the volumetric plane $\ln (1+e)$ vs. $\ln p$. They are denoted as normal compression lines, critical state line and normal extension lines. The maximum angle of $\psi_{\dot{\varepsilon}}$, for which the asymptotic states could reasonably be studied, was $\psi_{\dot{\varepsilon}}=110^{\circ}$. For higher angles, asymptotic state would have been reached at extremely low stresses (below 
$0.5 \mathrm{kPa}$ ), where the results were scattered and unreliable. We thus could not confirm the existence of the limit state denoted as $\pm d$ in Figure 2(a).

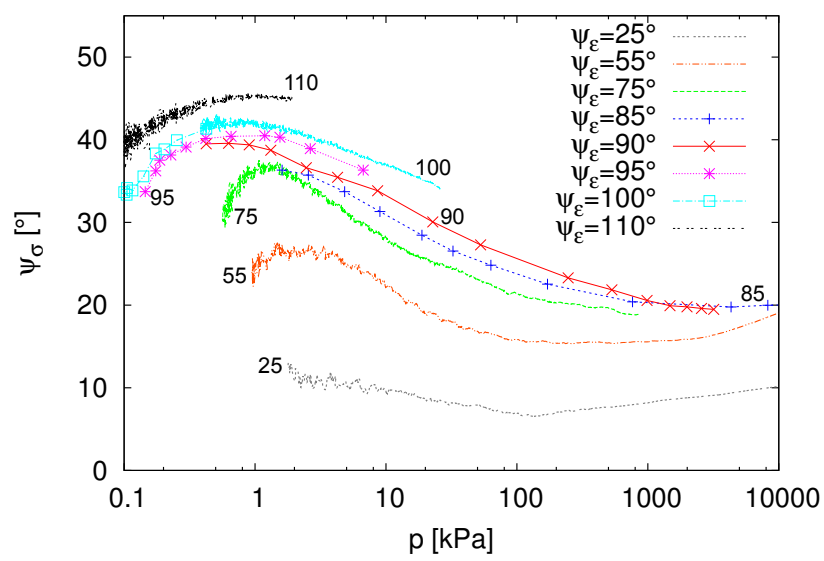

(a)

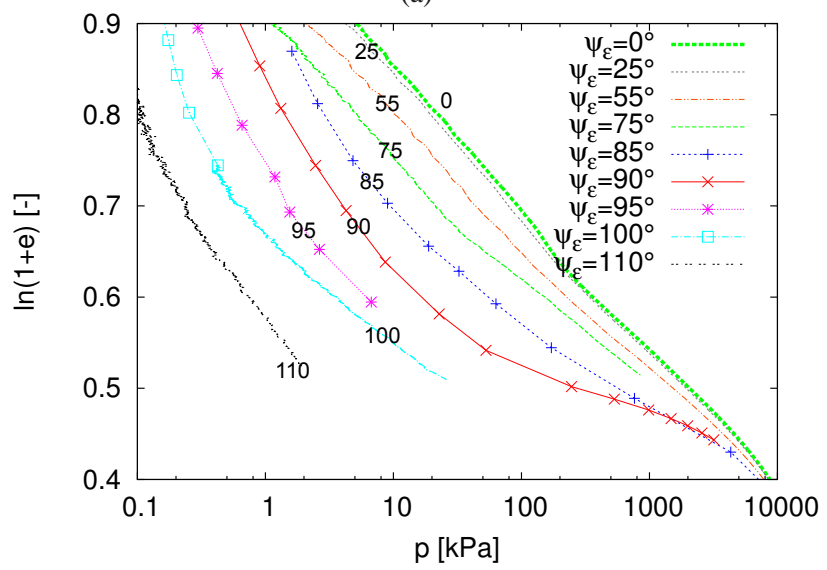

(b)

Fig. 8 Asymptotic states of an assembly of spherical particles, summary of the DEM simulations.

\section{Conclusions}

In the paper, we presented an investigation of the asymptotic properties of granular assemblies. The concept was first introduced, following description of an extensive DEM study. Although the particles were elastic (non-crushable), the asymptotic behaviour was clearly observed. Asymptotic behaviour thus appeared to be an inherent property of the granular assembly, caused primarily by particle rearrangement. The 
simulations indicated the existence of extension asymptotic states and normal extension lines.

Acknowledgements Financial support by the research grants GACR P105/12/1705 and TACR TA01031840 is greatly appreciated.

\section{References}

[1] Casagrande, A.: Characteristics of cohesionless soils affecting the stability of slopes and earth fills. Journal of the Boston Society of Civil Engineers 23(Jan.), 257-276 (1936)

[2] Cundall, P.A., Strack, O.D.L.: A discrete numerical model for granular assemblies. Géotechnique 29(1), 47-65 (1979)

[3] Gudehus, G.: Physical Soil Mechanics. Springer, Berlin (2011)

[4] Gudehus, G., Mašín, D.: Graphical representation of constitutive equations. Géotechnique 59(2), 147-151 (2009)

[5] Gudehus, G., Goldscheider, M., Winter, H.: Mechanical properties of sand and clay and numerical intergration methods: some sources of errors and bounds of accuracy. In: Gudehus, G. (ed.) Finite Elements in Geomechanics. pp. 121-150. Wiley, Chichester (1977)

[6] Jop, P., Forterre, Y., Pouliquen, O.: A constitutive law for dense granular flows. Nature 441, 727-730 (2006)

[7] Mašín, D.: Asymptotic behaviour of granular materials. Granular Matter (submitted) (2012)

[8] Schofield, A.N., Wroth, C.P.: Critical State Soil Mechanics. McGraw-Hill, London (1968)

[9] Šmilauer, V., Catalano, E., Chareyre, B., Dorofenko, S., Duriez, J., Gladky, A., Kozicki, J., Modenese, C., Scholtès, L., Sibille, L., Stránskỳ, J., Thoeni, K.: Yade Documentation. The Yade Project, 1st edn. (2010), http://yadedem.org/doc/ 